\title{
A semitransparent snake-like tactile and olfactory bionic sensor with reversibly stretchable properties
}

\author{
Guofa Cai, Jiangxin Wang, Meng-Fang Lin, Jingwei Chen, Mengqi Cui, Kai Qian, Shaohui Li, Peng Cui \\ and Pooi See Lee
}

\begin{abstract}
Many organisms and animals have sensing abilities that are different from those of human beings; for example, snakes have strong smell-, vibration-, touch- and heat-sensing abilities. A nature-mimicking sensing platform capable of sensing multiple stimuli, such as strain, pressure, temperature and other uncorrelated conditions, is highly desirable to broaden the applications of sensors. Here, we construct a semitransparent intelligent skin-like sensing platform based on polyaniline (PANI) nanowire arrays that can act as a bionic component by simultaneously sensing tactile stimuli and detecting colorless, odorless gas. Our multifunctional bionic sensing strategy is remarkably adaptive for versatile applications. The strain-sensing performance is superior to that of most conducting polymer-based sensors reported so far and is comparable to or even better than traditional metal and carbon nanowire/nanotube-based strain sensors. The highest gauge factor demonstrated is 149 , making our system a remarkable candidate for strain-sensing applications. The sensor can accurately detect a wide range of human motions. We also demonstrate the simultaneous controlled olfaction ability for the detection of methane with high sensitivity and a fast response time. These results enable the realization of multifunctional and uncorrelated sensing capabilities, which will afford a wide range of applications to augment robotics, treatment, simulated skin, health monitoring and bionic systems.
\end{abstract}

NPG Asia Materials (2017) 9, e437; doi:10.1038/am.2017.181; published online 13 October 2017

\section{INTRODUCTION}

Stretchable and wearable electronics have been extensively investigated for a broad range of applications, including electronic skins, flexible displays, health-monitoring devices and energy-harvesting devices. ${ }^{1-4}$ Breakthroughs have been made in the theoretical and practical aspects of these related technologies. Meanwhile, it is highly attractive to integrate more functionalities and novel features into one device for smart and multifunctional systems by mimicking complex biological systems. Among the various complex biological systems, snakes are unique legless reptiles with elongated bodies covered in overlapping scales. Most snakes use stretchable belly scales to travel by gripping surfaces and have strong olfactory capabilities to track prey. The skin and olfactory organ can collect external stimuli and output bioelectrical signals to the nervous system/brain. In practical applications, artificial e-skin capable of sensing multiple stimuli, such as strain, pressure, vibration, temperature and other uncorrelated conditions, is highly desirable. Despite the promise of these systems, only limited successful examples of stretchable sensors that perceive force and chemical stimuli have been reported. ${ }^{5-7}$

To achieve high-performance artificial e-skin, the directional design and fabrication of conductive sensing channel materials are prerequisites. Tailored materials in the sensing channel with specific nanostructures can meet critical requirements such as enhanced sensitivity, stretchability and mechanical stability. Recently, a spider-inspired crack-based structure composed of a platinum layer on top of a viscoelastic polymer was proven to be an effective geometric patterning method for the preparation of a sensor system with ultrahigh sensitivity. ${ }^{8}$ Recently, a variety of sensing systems with high sensitivity based on metal or inorganic active channels in elastomer composites have been prepared to recognize human activities. However, most of the reported sensors are of single functionality, incompatible to the sensing of multiple external stimuli and thus unfavorably hinder the simultaneous detection of complex stimuli. ${ }^{9,10}$ In addition, complex transfer steps or patterning steps on an elastic substrate are needed to fabricate stretchable structures. Good adhesion between the substrate and metal or inorganic sensing channel layers is difficult to achieve, which could lead to device breakdown under repeated large deformations with consequent fatigue accumulation due to their mechanical mismatch. ${ }^{11}$ Moreover, the high processing costs limit their practicality for devices that need to be disposable, low cost and scalable. Furthermore, optical transparency and low thickness is preferred in e-skin for aesthetic, optogenetic usage and comfort reasons because e-skin is usually attached to visible parts of the human body, such as the limbs, wrist or neck, and for smart garments in wearables and interactive soft robotics. Recently, efforts on semiconducting polymeric nanowires for stretchable and transparent devices were demonstrated by some research groups. Cho and coworkers $^{12}$ demonstrated a stretchable and transparent organic semiconducting layer via blending self-assembled poly(3-hexylthiophene) nanowires with an elastomeric polymer for use in stretchable organic thin-film transistors. The transistors show superior and reliable stretching stability during stretching at $100 \%$ strains. 
Polyaniline (PANI) is one of the most promising conducting polymers with many advantages, such as inherent flexibility, enhanced conductivity, good environmental stability, cost-effective fabrication and good scalability. Moreover, PANI is capable of sensing multiple stimuli, including biological, chemical, gas, temperature, humidity, strain and pressure, owing to its unique and controllable chemical and electrical properties. ${ }^{13-18}$ Unfortunately, the typical formation of PANI thin films, based on spin or spray processes, is unable to produce stretchability because of the chain rigidity of PANI. Inspired by the scale-structured stretchable skin and sophisticated olfactory ability of snakes, a multifunctional electronic skin sensor is designed via the growth of bilayer PANI with a planar particulate film and vertically aligned nanowires on a thin polydimethylsiloxane (PDMS) substrate. The unique snake scale-like stretchable structures result from the combination of out-of-plane nanowire arrays and formed ridges and endow the sensor with high sensitivity and excellent mechanical conformability. The sensing system is capable of not only monitoring stretching strains but also detecting odorless and explosive $\mathrm{CH}_{4}$ gas with high sensitivity. This unconventional olfactory chemoreceptordecorated skin structure paves the way to the realization of uncorrelated multifunctional artificial skin. This biomimicry-sensing platform could find broad applications in artificial intelligence, humanmachine interfaces and robotics, specifically in bionic systems.

\section{EXPERIMENTAL PROCEDURES}

\section{Materials}

Aniline ( $\geqslant 99.5 \%)$, perchloric acid $(70 \%)$, ammonium persulfate and hexane (anhydrous, 95\%) were purchased from Sigma-Aldrich (Singapore). The silicone elastomer base and curing agent (SYLGARD 184) were purchased from Dow Corning Corporation (Auburn, MI, USA). Copper tape was purchased from 3M (Singapore). Silver paste was purchased from Ted Pella, Inc (Redding, CA, USA). All reagents were used as received without further purification.

\section{Preparation of thin PDMS}

The thin PDMS elastomer was prepared by mixing the silicone elastomer base and the curing agent with a ratio of 10:1. Then, the liquid mixture was diluted with hexane to form a $10 \mathrm{wt} \%$ solution. Then, the diluted liquid mixture was transferred onto a watch glass. The thin PDMS elastomer was prepared after degassing and thermally curing the diluted liquid mixture at $60{ }^{\circ} \mathrm{C}$ for $2 \mathrm{~h}$. Hexane was evaporated during the PDMS curing process. Here, PDMS with a thickness of approximately $150 \mu \mathrm{m}$ (Supplementary Figure S1) was prepared and used as the elastic substrate.

\section{Preparation of stretchable PANI nanowire arrays on a thin PDMS elastic substrate}

PANI nanowire arrays were in situ deposited on the surface of the thin PDMS substrate through chemical bath deposition. ${ }^{19}$ Typically, the solution for chemical bath deposition contained $1 \mathrm{M} \quad\left[\mathrm{HClO}_{4}\right], 0.01 \mathrm{M}$ aniline and $0.0067 \mathrm{M}$ ammonium persulfate. The as-obtained thin PDMS layer was used as the substrate and was vertically supported on the wall of the bath container at $0{ }^{\circ} \mathrm{C}$ (ice bath) for $24 \mathrm{~h}$ to deposit the PANI nanowire arrays. After depositing, the samples were rinsed with de-ionized water and finally dried naturally. The functional PANI nanowire array channel was directly grown on the thin PDMS substrate, and a highly stretchable device was obtained.

\section{Preparation of the bionic snake skin sensing platform}

The sensing platform was assembled by employing the PANI nanowire arraycoated PDMS as the conductor and elastomeric substrate. For strain- and gassensing applications, copper tape was connected at the two ends of the PANI nanowire arrays. Silver paste was used to minimize the contact resistance. To prepare the pressure sensor, two PANI nanowire array-coated PDMS films were brought into face-to-face contact to produce an Ohmic-like contact. Then, the terminal regions were connected with copper tape to form stable electrical connections. Finally, the device was sealed by a thermal laminator to ensure long-term stability.

\section{Characterization}

The transmittance of the sensor was detected using a SHIMADZU UV-3600 spectrophotometer (Shimadzu, Kyoto, Japan). Fourier transform infrared spectroscopy (FTIR) was performed using a Spectrum GX FTIR spectrometer (PerkinElmer Inc., Waltham, MA, USA). To illustrate the gas-sensing mechanism of PANI, Fourier transform infrared-attenuated total reflectance (FTIRATR) spectra were measured for the PANI nanowire before and after exposure to the reducing gas $\mathrm{CH}_{4}$. FTIR-ATR can be used to rapidly analyze the characteristics of PANI nanowires after exposure to the reducing gas $\mathrm{CH}_{4}$. After exposure to $1 \% \mathrm{CH}_{4}$ gas, the samples were subjected to FTIR-ATR analysis within $30 \mathrm{~s}$. The microstructures of the PANI nanowire arrays were characterized by scanning electron microscopy (SEM) (JEOL 7600F, JEOL, Tokyo, Japan). Dynamic mechanical analysis was performed on a universal testing machine (DMA Q800, TA Instruments, New Castle, DE, USA). For the stretching tests, the sensor was fixed on home-built stretching stage to apply different strains. The frequency tests for the sensor were performed in the range of $0.025-075 \mathrm{~Hz}$ under an applied strain of $0-20 \%$. For human motion detection, the sensor was attached to face skin using double-sided tape or mounted on gloves. $\mathrm{CH}_{4}$ gas-sensing experiments were conducted in a gas chamber using two terminal devices at room temperature. $\mathrm{N}_{2}$ gas was employed as a carrier gas to dilute the test $\mathrm{CH}_{4}$ gas and to purge the devices after stopping the test gas feed. The flow rate of the carrier gas was kept at a constant $5 \mathrm{sccm}$, and the $\mathrm{CH}_{4}$ gas source had a concentration of 1\% (10 000 p.p.m.) in pure nitrogen. All resistance and current changes were measured using a Keithley analyzer (Model 4200, Keithley Instruments, Cleveland, OH, USA).

\section{RESULTS AND DISCUSSION}

\section{Microstructure characteristics}

The deposition process of PANI nanowire arrays is illustrated in Figure 1a. In the chemical oxidation polymerization process of PANI, the bulk solution and solid substrates are two possible competing nucleation sites for the growth of PANI. Heterogeneous nucleation occurs on the solid PDMS substrates when dilute aniline $(\leqslant 0.05 \mathrm{M})$ is used. ${ }^{20}$ Most of the active nucleation centers are generated on PDMS substrates at a comparatively fast rate in the initial stage of polymerization, forming a planar PANI thin film on the PDMS substrate. The active nucleation centers are isolated from each other in the early stage because of the hydrophobic nature of the PDMS substrate and the low concentration of aniline, which was previously proven by an in situ technique. ${ }^{21}$ Homogeneous nucleation and growth in the solution occur simultaneously and cause precipitation onto the active sites on the thin film. With depleted reactive aniline cation radicals and oligomeric intermediates, the vertical growth of PANI nanofibers was achieved on the PANI polymerized solid PDMS substrates. Therefore, a bilayer composed of planar PANI and aligned nanowires on the PDMS elastic substrate was obtained. ${ }^{19,20}$ SEM images of PANI on the PDMS elastic substrate are shown in Figure 1b. PANI nanowire islands and cracks in the film were observed in the low-magnification image. In addition, suspended PANI bundles bridging the gaps between the nanowire islands were also observed, which are critical to maintain interconnections in the film. On the other hand, the PANI islands act as anchors to prevent film detachment from the elastic substrate. In the top-view SEM image of the PANI nanowire film, the bright white spots correspond to the tips of the aligned PANI nanowires. The inset of Figure $1 \mathrm{~b}$ shows the high-magnification SEM image of the tilted nanowires with diameters ranging from 35 to $60 \mathrm{~nm}$. The lengths of the nanowires range from 100 to $250 \mathrm{~nm}$ (Supplementary Figure S2). The PANI nanowire arrays exhibit an optical transmission of approximately $60 \%$ within the wavelength range of $495-570 \mathrm{~nm}$, producing green color PANI 
nanowire arrays (Figure 1c). The transmission wavelength of the PANI nanowire arrays is similar to that of a PANI thin film prepared by the layer-by-layer self-assembly method. ${ }^{22}$ The insets of Figure 1c show photographs of the as-grown PANI nanowire arrays on the PDMS elastic substrate. The green color of the obtained sample provides preliminary evidence of the formation of PANI in its conducting emeraldine salt form. ${ }^{23}$ To further investigate the oxidative state of PANI, FTIR was conducted on a Spectrum GX FTIR spectrometer, and the results are shown in Figure 1d. The peaks located at 1569 and $1484 \mathrm{~cm}^{-1}$ correspond to the quinoid and benzenoid ring-stretching vibrations. The appearance of the peak at $1373 \mathrm{~cm}^{-1}$ is assigned to C$\mathrm{N}$ stretching vibrations in the adjacent quinonoid or quinonediimine structures. The peak at $1294 \mathrm{~cm}^{-1}$ is attributed to C-N stretching vibrations characteristic of the secondary aromatic amine. $\mathrm{C}-\mathrm{H}$ inplane bending vibrations are usually observed in the range of 1010$1170 \mathrm{~cm}^{-1}$. The band at $1238 \mathrm{~cm}^{-1}$ is characteristic of conducting protonated PANI and corresponds to a $\mathrm{C}-\mathrm{N}^{+\cdot}$ stretching vibration in the polaron structure. ${ }^{24} \mathrm{C}-\mathrm{H}$ out-of-plane bending vibrations are reflected by the peaks at 793 and $495 \mathrm{~cm}^{-1}$. The peak observed at $618 \mathrm{~cm}^{-1}$ is ascribed to the $\mathrm{ClO}_{4}{ }^{-}$ion. ${ }^{25}$ These characteristic bands confirm that PANI is in the conducting emeraldine salt phase.

\section{Tactile capacity evaluation of the PANI nanowire array-based} sensing platform

To evaluate the tactile capacity and stretchability of the PANI nanowire array-based sensing platform, dynamic mechanical analysis was performed. The stress and strain profiles show that coating of PANI on PDMS had no significant influence on the mechanical properties (Figure 2a). Both films have almost the same Young's modulus (approximately $0.65 \mathrm{MPa}$ ), and both can withstand over
$190 \%$ strain. The current responses with an applied voltage of $0-1 \mathrm{~V}$ were measured under $0-100 \%$ strain for the PANI nanowire arraybased strain sensor with a size of $0.5 \times 1 \mathrm{~cm}^{2}$. The PANI nanowire array-based strain sensor demonstrated linear $I-V$ curves under various strains (Supplementary Figure S3), indicating the Ohmic behavior of the sensor. The current monotonically decreased with increased tensile strain. The relative resistance changes $\left(\left(R-R_{0}\right) /\right.$ $R_{0}=\Delta R / R_{0}$, where $R$ is the resistance under stretching and $R_{0}$ is the resistance at $0 \%$ strain) with respect to strain, calculated from the $I-V$ curves, are shown in Figure 2b. The relative resistance change increased when a larger strain was applied. The PANI strain sensor shows a large relative resistance change of $14897 \%$ at $100 \%$ strain. The increased number of ridges and islands upon the application of strain is the main cause of the piezoresistive effects of the sensor. A reversible, large resistance change is highly desirable for strainsensing applications and is a prerequisite for high sensitivity. Figures $2 \mathrm{c}-\mathrm{e}$ and Supplementary Figure $\mathrm{S} 4$ show the strain-induced resistance changes as the strain was increased from 0.2 to $60 \%$. The sensor shows reproducible and reliable responses even under small strain $(0.2 \%)$. These results illustrate that the PANI nanowire arraybased strain sensor works well over small to large strain. Supplementary Figure S5 depicts the relative resistance changes with respect to strain under 10 and $60 \%$ stretch-release cycles with a moving stage speed of $0.05 \mathrm{~mm} \mathrm{~s}^{-1}$. Slight hysteresis is observed during $60 \%$ stretch-release cycling because of the mechanical properties of PDMS. However, the original resistance of the sensor is almost fully recovered after releasing the strain, and the hysteresis is negligible within $10 \%$ strain, indicating that our PANI-based strain sensor possesses outstanding signal recovery. In addition, the sensor also has important advantages of low creep and fast response. As seen in

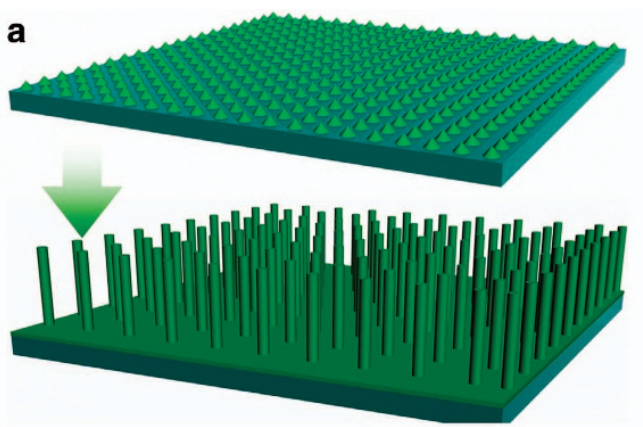

C

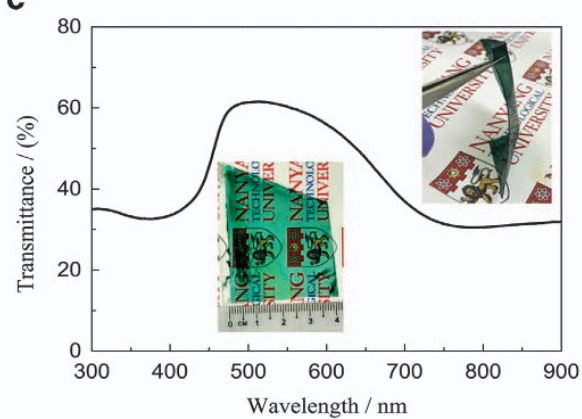

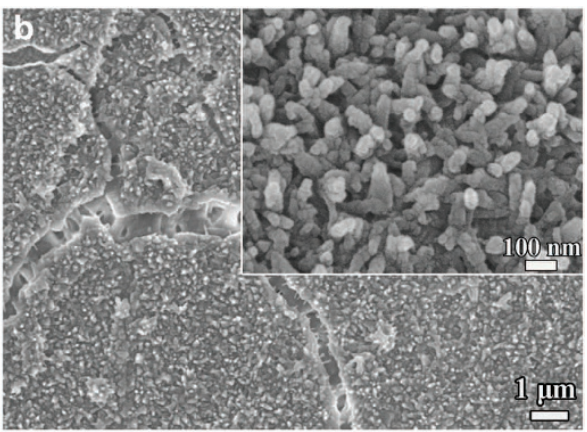

d

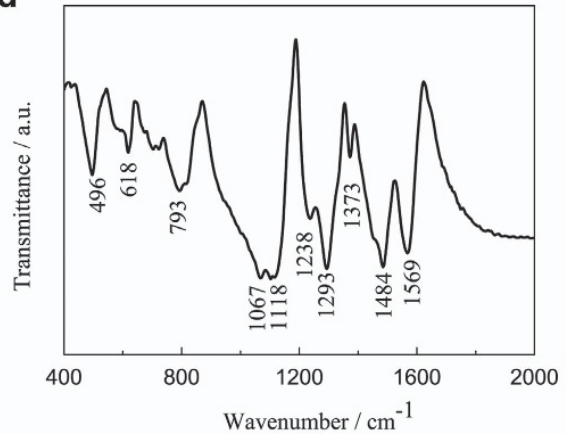

Figure 1 Chemical and microscopic structure of the PANI nanowire arrays. (a) Formation process of the PANI nanowire arrays. (b) SEM images of the PANI nanowire arrays on a PDMS substrate; the inset presents the high-magnification SEM image of the titled nanowire. (c) Transmittance spectrum of the PANI nanowire arrays on a PDMS substrate over the wavelength range of 300-900 nm; the inset presents photographs of the skin-like film. (d) FTIR spectrum of PANI powder scratched from the PDMS substrate. 
Supplementary Figure S6, a step strain of $10 \%$ was imposed within $1 \mathrm{~s}$, exhibiting a percent overshoot and creep recovery time of $16.5 \%$ and $7.5 \mathrm{~s}$, respectively. The gauge factor represents the sensitivity of the sensors and can be defined as $\left(\Delta R / R_{0}\right) / \varepsilon$, where $\varepsilon$ is the applied strain. The highest gauge factor achieved in this work is 149 at $100 \%$ strain (Supplementary Figure S7). The strain sensor can simultaneously achieve excellent sensitivity, stretchability and linearity, indicating that the PANI nanowire arrays are excellent candidates for strain-sensing applications. The nanowire array-based sensor outperformed conventional metal strain sensors in terms of both strain range and gauge factor $(\varepsilon<5 \%$, gauge factor $\approx 2),{ }^{26}$ as well as most of the recently reported strain sensors based on carbon nanotubes $(0.82$ between 0 and $40 \%$ strain or $10-25$ between 0 and $550 \%$ strain), ${ }^{27}$ graphene (7.1 at $100 \%$ strain), ${ }^{28}$ silver nanowire (14 at $30 \%$ strain $),{ }^{29}$ hydrogel $(1.51$ at $1000 \%$ strain $)^{30}$ and ionic conductive fluid $(0.348 \pm 0.11$ at $700 \%$ strain); ${ }^{31}$ furthermore, the gauge factor is nearly 180 times higher than that recently reported for PANI nanowires. ${ }^{16}$ Moreover, our PANI nanowire array-based sensor also showed a precise response independent of the test frequency $(0.025-0.75 \mathrm{~Hz})$ or strain rate under the application of $20 \%$ stretch-release strain cycles (Figure 2f). The stability of the sensor was also investigated by applying 2000 stretch-release cycles at $20 \%$ and $60 \%$ strain, at which the strain gauge factors are approximately 15 and 45 . The resistances were collected at the repeated strain states (Supplementary Figure S8). The resistance of the sensor remains almost constant with minor fluctuations within the first 900 cycles for both strain states. Nevertheless, the resistance decreases slightly after 900 cycles at $20 \%$ strain and then remains almost constant up to 2000 cycles. At the large strain state (60\% strain), the resistance increases with increasing cycle number after 900 cycles, and the value is 1.38 times larger than its initial value after 2000 cycles. Overall, the sensor shows large fluctuations in the signal at large strain cycles. Photographs of the sensor stretched to different strains are shown in Supplementary Figure S9.

To understand the underlying mechanism of the device stretchability, the microstructural changes of the same PDMS/PANI film under different levels of strain were examined by SEM, as shown in Figures $3 \mathrm{a}-\mathrm{c}$. The relaxed states after recovery from different strain states were also examined by SEM, as shown in Figures $3 \mathrm{e}$ and $\mathrm{f}$. It can be seen that the PANI nanowire islands and ridges (or cracks) are analogous to the scales that cover a snake's skin, and thus the microstructure and stretchability of our device resemble a snake's skin (inset of Figure 3d). The isolated PANI islands, crack density and crack width increased with increased tensile strain. In addition, the ridges are predominantly perpendicular to the tensile direction. Despite the appearance of more islands and cracks under strain, many lateral PANI nanofibers remained in the gap between the islands in the $40 \%$ strain state, as indicated in the inset of Figure $3 \mathrm{~b}$ (with arrows), and most of the PANI nanowire islands were still interconnected even in the $80 \%$ strain state (Figure 3c). Moreover, the size of the PANI nanowire islands also increased with increased tensile strain after careful comparison of the images of the 80 and $40 \%$ strain states. This result could be attributed to the PDMS elastic substrate that was firmly adhered to the PANI nanowire arrays and assisted in the delocalization of the strain, which prevented cracks from expanding into channel cracks. Remarkably, the cracks caused by strain were self-repaired after full strain recovery from both the 40 and $80 \%$ strain states (Figures $3 \mathrm{e}$ and $\mathrm{f}$ ), and there was no significant difference compared with the original state (Figure $3 \mathrm{~d}$ ). The strain was accommodated by the reversible opening and closing of the ridges (or cracks) during the corresponding stretched and relaxed states. The resistance changes in the sensor under different strains were also demonstrated on a complete circuit composed of an LED indicator
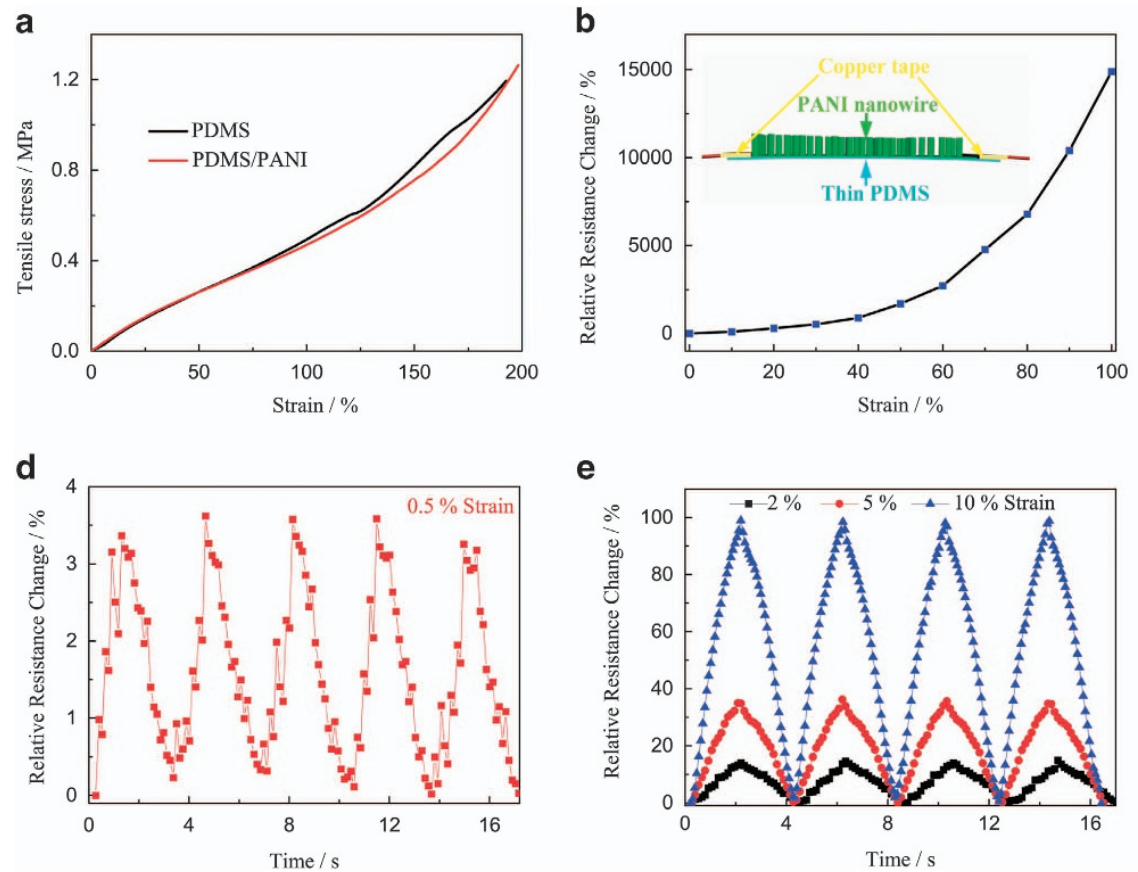

e

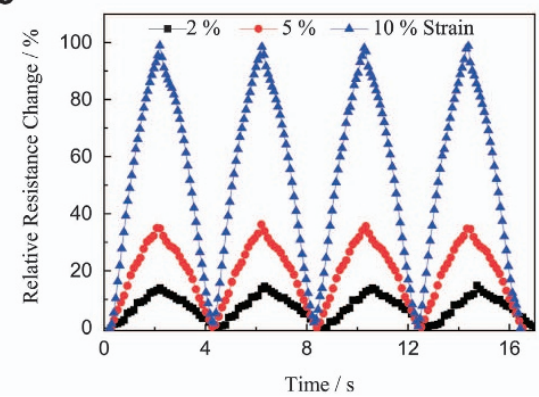

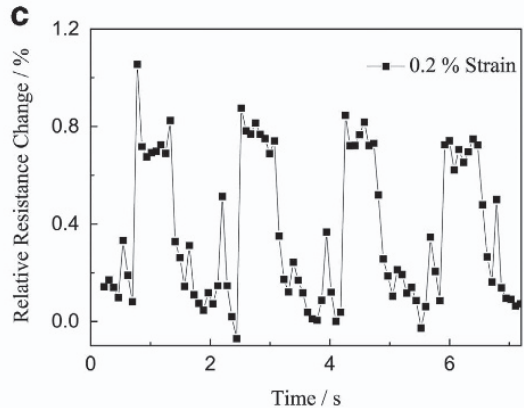

f

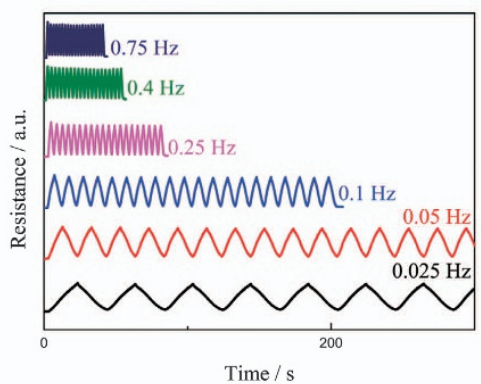

Figure 2 The performance of the PANI nanowire array-based strain sensor. (a) Tensile stress-strain profile of the PDMS and PDMS/PANI films. (b) Plots of the relative resistance change versus strain for the PANI nanowire array-based sensor; the inset is a schematic illustration of the structure of the sensor. Relative resistance changes as a function of time under different strains of (c) $0.2 \%$ strain, (d) $0.5 \%$ strain and (e) 2 , 5 and $10 \%$ strain. (f) Relative resistance changes at different frequencies at $20 \%$ strain. 
with a PANI nanowire array-based strain sensor as the conductor, as shown in Figures 3g1-g4. The LED indicator was successfully lit at various strains (the driving voltage was $5 \mathrm{~V}$ ). The luminance of the LED indicator decreased with increasing tensile strain (with a strain rate of $1 \mathrm{~mm} \mathrm{~s}^{-1}$ ), which corresponds to the increased resistance of the sensor with increased tensile strain.

To demonstrate the capability of our semitransparent, highly stretchable, and sensitive sensor as a wearable strain sensor for the full-range detection of human motions, the sensor was directly attached on the forehead to detect the weak muscle motions caused by respiration, eye movement or speaking. The sensor was also mounted on a glove to monitor the large-scale bending and stretching of a human finger knuckle. The facial muscle on our forehead is stretched when we deeply inhale while breathing. As shown in Figure $4 \mathrm{a}$, the stretching or compression movement of the human forehead muscle generated by deep breathing and eye movement could be clearly detected by our strain sensor with high signal-to-noise ratios and highly repeatable patterns. This facial muscle is compressed when we close our eyes, and this is reflected by the drop-in resistance in Figure 4b. The sharp and rapid response of the sensor was also observed during rapid eye blinking. Moreover, complex epidermis/ muscle movements on the human forehead associated with the pronunciation of some words, such as 'Hi', 'Hello', the Chinese word 'Ni Hao' and the material names 'polyaniline' and 'nanowire'. Each word could be monitored by the sensor with remarkably characteristic patterns and good repeatability. It can be seen that the waveform of

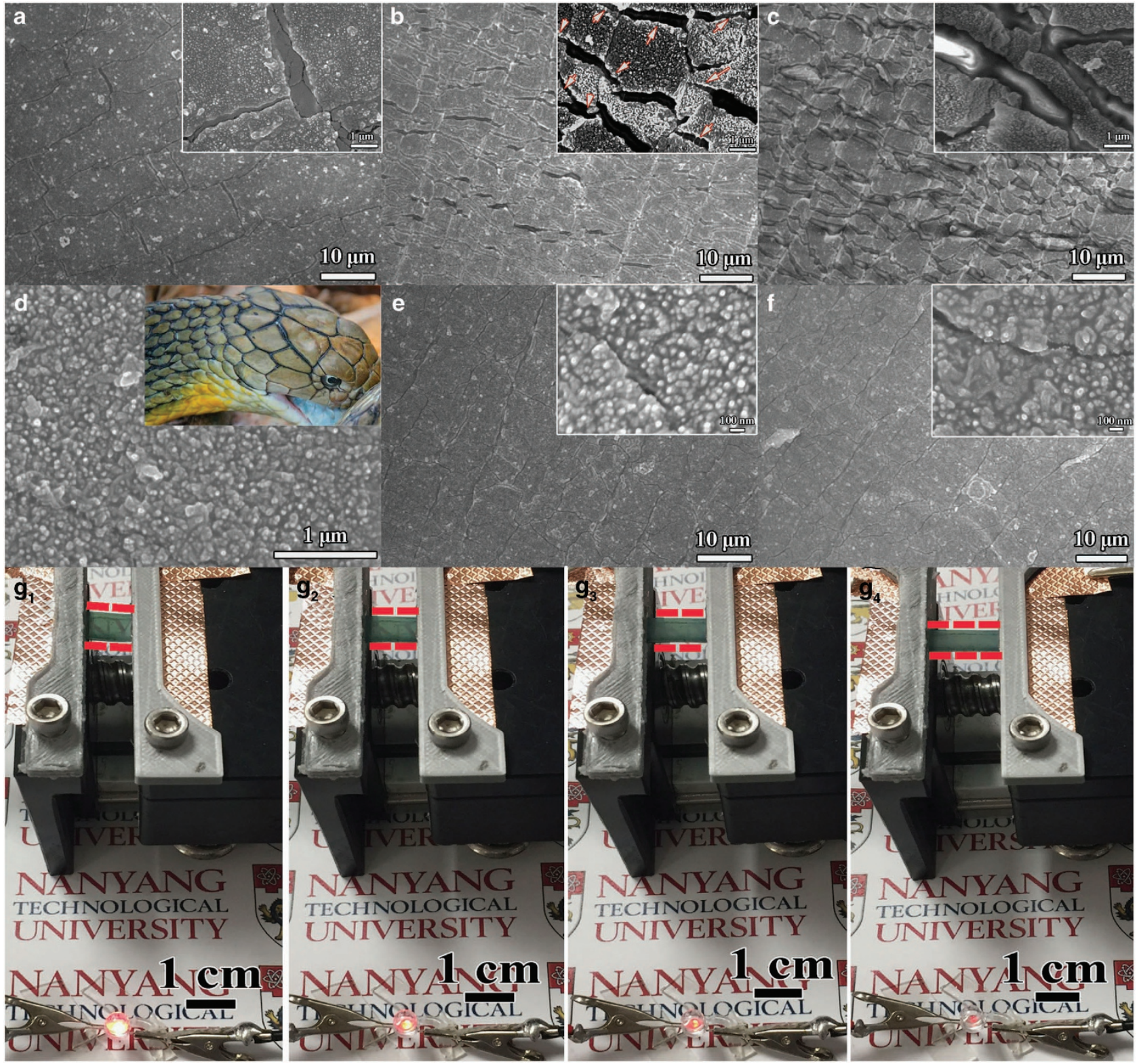

Figure 3 Stretching mechanism of the film. SEM images of the PANI nanowire array film under different strains of (a) $0 \%$, (b) $40 \%$ and (c) $80 \%$. Highmagnification SEM images of the nanowire islands (d) in the 0\% strain state, (e) fully recovered from the $40 \%$ strain state and (f) in the $80 \%$ strain state. (g) Photographs of a powered LED indicator integrated with the PANI nanowire arrays on a PDMS substrate under different tensile strains of $\left(\mathbf{g}_{1}\right) 0 \%$, $\left(\mathbf{g}_{2}\right)$ $25 \%,\left(\mathbf{g}_{3}\right) 50 \%$ and $\left(\mathbf{g}_{4}\right) 75 \%$; the sensor is indicated the red dashed line. 
each word was apparently different, and the repeatability was good (Figure 4c). Furthermore, our sensor also showed a sharp and rapid response to the high degree bending during finger movements (Figure 4d). During this large-scale bending and stretching test, the sensors exhibited overshooting in response to acceleration during motion. The peaks associated with acceleration are generated by the low creep of the strain sensor. A similar phenomenon was observed in other strain sensors based on silicone rubber/carbon materials. ${ }^{32}$ The results indicate the high sensitivity and broad range of the sensor in sensing strain, as well as the capability of detecting multiple deformations. Moreover, the PANI nanowire arrays also exhibit pressuresensing behavior when the sensor is assembled from two PANI nanowire array films with a face-to-face construction (Supplementary Figure S10a). The pressure-sensing mechanism is due to the forcedependent bridging contacts between the PANI nanowires and the interlocking of the nanowire arrays. The number of bridged PANI nanowires depends on the applied external force. A small compressive deformation of the soft device caused a substantial number of PANI nanowires to come in contact under the application of pressure, leading to additional conductive pathways. When a voltage of $1 \mathrm{~V}$ was applied to the sensor, an increased current was observed after loading the pressure. On unloading, the sensor recovered to its original shape, reducing the amount of interlocking PANI nanowires and therefore leading to a decreased current. The relative resistance changes were rapidly detectable with the loading and unloading of a pressure of $8 \mathrm{kPa}$, which is similar to the pressure induced by a human finger touch $(<10 \mathrm{kPa}) .^{33}$ We can define the gauge factor for this pressure sensor as $\left(\Delta R / R_{0}\right) / \Delta P$, where $\Delta P$ is the pressure change. The gauge factor is approximately $7.5 \times 10^{-3} \mathrm{kPa}^{-1}$, which is comparable to that of typical pressure sensors $\left(5-8 \times 10^{-3} \mathrm{kPa}^{-1}\right){ }^{34}$ Therefore, a finger touch can be accurately detected by the PANI nanowire array-based pressure sensor (Supplementary Figure S10b). Thus, the sensor is able to detect various human activities and is suitable for tactile wearable devices.

\section{Olfactory capacity evaluation of the PANI nanowire array-based sensing platform}

The above results demonstrate that the PANI nanowire arrays on a PDMS elastic substrate exhibit excellent performance as a wearable tactile sensor. In addition, we also demonstrate that these PANI nanowire arrays on a PDMS elastic substrate can deliver outstanding properties as a stretchable smelling skin. To date, various $\mathrm{CH}_{4}$ gas sensors have been reported. ${ }^{35,36}$ However, to the best of our knowledge, there is no report dedicated to stretchable $\mathrm{CH}_{4}$ gas sensors. Here, the hazardous gas $\mathrm{CH}_{4}$ was applied as the target gas to evaluate the gas-sensing performance of the stretchable PANI films. The highly volatile $\mathrm{CH}_{4}$ gas is a colorless and odorless gas that can accumulate in the walls of coal mines and be released during mining. Methane can accumulate and cause lethal explosions if not monitored. If inhaled by humans or animals, $\mathrm{CH}_{4}$ molecules can effectively replace oxygen molecules in the body, causing suffocation. ${ }^{37}$ PANI nanowires as the sensing layer to detect $\mathrm{CH}_{4}$ gas has many advantages, such as low cost, light weight, ease of synthesis and high sensitivity at room temperature. It can be seen that the conductivity of the sensors decreased when exposed to $\mathrm{CH}_{4}$ gas and then gradually increased when re-exposed to air (Figure 5a). As is well known, PANI is conductive in acid or in its doped emeraldine salt form and insulating in its emeraldine base or dedoped form. FTIR-ATR spectra were measured to illustrate the gas-

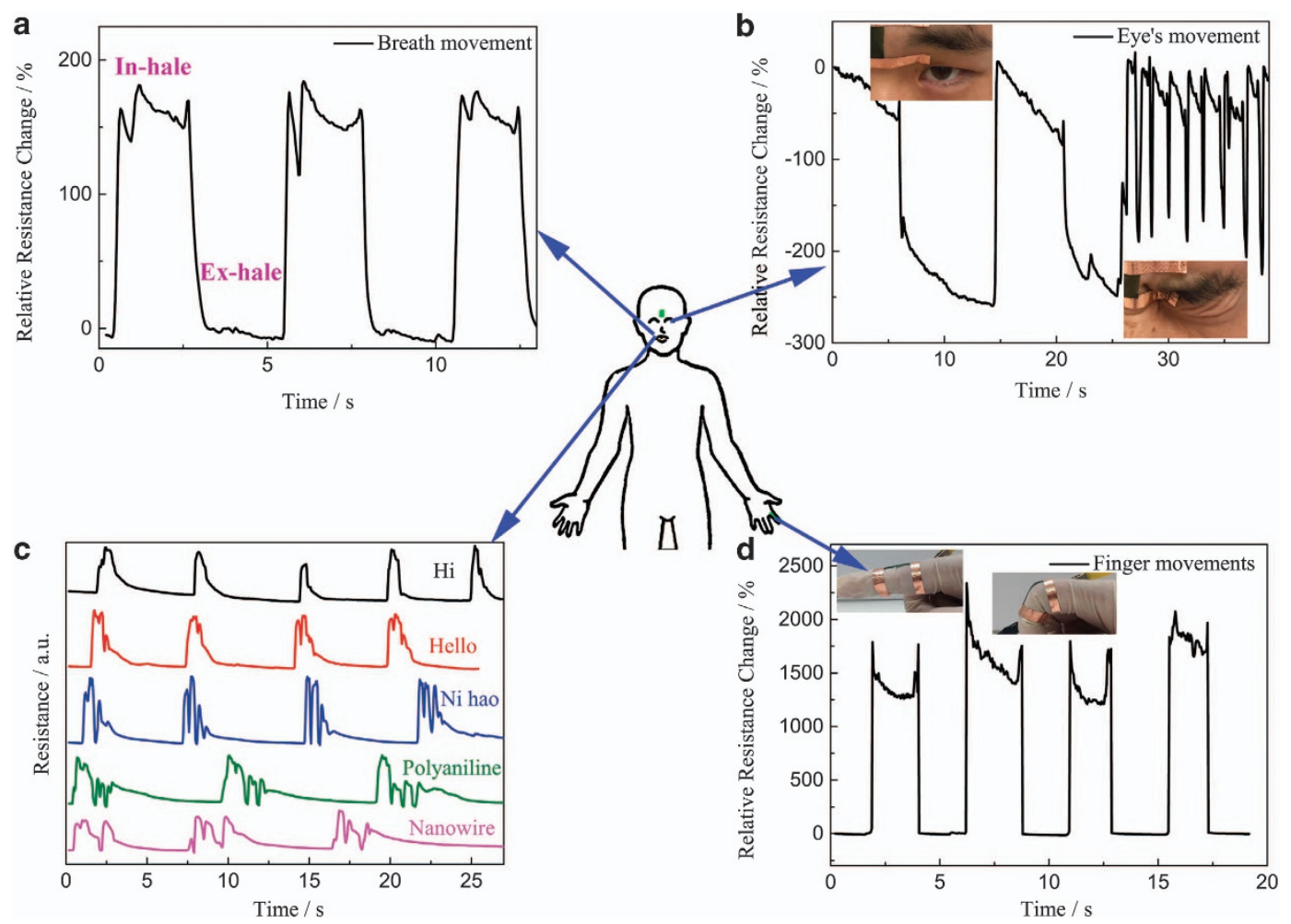

Figure 4 Monitoring of various human motions in real time. (a) Relative resistance changes versus time caused by respiration. (b) Relative resistance changes versus time caused by eye movement; the inset shows the wearable sensor attached to the forehead for monitoring eye movement. (c) Relative resistance changes versus time caused by speaking. (d) Relative resistance changes versus time for large-scale bending and stretching of the finger knuckle; the inset shows the wearable sensor mounted on a glove for monitoring forefinger motion. 
sensing mechanism of PANI, as shown in Supplementary Figure S11. After exposure to the reducing gas $\mathrm{CH}_{4}$, the peaks located at $1002 \mathrm{~cm}$ -1 (C-H bending vibration), $1242 \mathrm{~cm}^{-1}\left(\mathrm{C}-\mathrm{N}^{+}\right.$stretching vibration) and $1464 \mathrm{~cm}^{-1}$ (benzenoid) were slightly blueshifted to $1013 \mathrm{~cm}^{-1}$, $1248 \mathrm{~cm}^{-1}$ and $1473 \mathrm{~cm}^{-1}$, respectively. In addition, the peaks at $1519 \mathrm{~cm}^{-1}\left(\mathrm{~N}-\right.$ benzenoid-N) and $1594 \mathrm{~cm}^{-1}(\mathrm{~N}=$ quinoid $=\mathrm{N})$ were more obvious after exposure to the reducing gas $\mathrm{CH}_{4}$, indicating that deprotonation occurred and part of PANI changed from the conducting emeraldine salt form to the insulating emeraldine base form when exposed to the reducing gas $\mathrm{CH}_{4}$. This results in a loss of conductivity. In addition, PANI is a $\pi-\pi^{\star}$ conjugated system that will generate charge transfer when $\mathrm{CH}_{4}$ gas molecules are adsorbed on the PANI surfaces. The conductivity of the sensor will change because of the charge interaction between the $\pi$ electrons of PANI and the gas molecules. ${ }^{35}$ The response is fast, the conductivity change is significant, and the sensing response is more than eight times greater. The recovery process is slow when the $\mathrm{CH}_{4}$ gas is switched off because of the slow removal of $\mathrm{CH}_{4}$ molecules from polyaniline at room temperature. Therefore, polyaniline requires a longer time to recover its conductivity compared with the response process, and thus the stabilized platform is not obvious during the recovery process (a similar phenomenon was observed for a PANI fiber sensing of 500 p.p. m. ammonia). ${ }^{38}$ As shown in Supplementary Figure S12, the recovery process is mostly completed within $20 \mathrm{~s}$, at which the current becomes relatively stable and no obvious change was observed even when the recovery time was doubled. To study the sensing performance of the gas under stretching, the measurement was also performed under different strains while maintaining the same gas exposure conditions (fixed $\mathrm{CH}_{4}$ concentration and on-off time; Figures 5b-e). The sensitivity of the sensor increases upon stretching for $0-50 \%$ strain, and a rapid drop is observed upon further strain (70\%). The highest conductivity change of approximately 700 times was achieved at 50\% strain. We can define the response and recovery time of the sensor as the time required to reach a stable output current when exposed to $\mathrm{CH}_{4}$ or air. The response time is much shorter than the recovery time, and both were constant after several seconds (s) under different strains (Supplementary Figure S13). The fastest response speed is $0.8 \mathrm{~s}$ when exposed to $\mathrm{CH}_{4}$ under $10 \%$ strain, which is much faster than that of previously reported PANI-based sensors. ${ }^{6,39,40}$ The increased sensitivity under strain may originate from the exposure of fresh PANI surfaces when the sensor is stretched. In addition, the conductivity decreased when a larger strain was applied, which can also lead to a higher sensitivity under a less conductive state, and a similar phenomenon was observed in a polypyrrole-based sensor. ${ }^{41}$ The results indicate that the sensor with a resistance in the range of $\sim 5 \times 10^{4} \Omega$ under strain would provide better gas sensitivity. However, upon exposure to $\mathrm{CH}_{4}$ gas, the sensor resistance under $70 \%$ strain becomes too large $\left(>10^{3} \mathrm{M} \Omega\right)$ to be accurately recorded by the equipment. After releasing the sensor from strain, almost the same sensitivity was observed as that before stretching (Supplementary Figure S14), illustrating the good reversibility of the sensor. In addition, the deprotonation/protonation of PANI can reversibly occur in the response/recovery processes, respectively, indicating that the sensor has good stability and repeatability (Supplementary Figure S15). Figure $5 f$ shows a much higher resistance change in the sensor under different strains when exposed to $\mathrm{CH}_{4}$ gas compared with stretching in air. The reduced physical contact between PANI ridges (or cracks) is the main cause of the resistance increase during the different stretched states in air. When the sensor is stretched in the presence of $\mathrm{CH}_{4}$ gas, chemical deprotonation occurs, which is the dominating factor leading to the dramatic resistance change. Moreover, the selectivity of the PANI nanowire array-based sensor was investigated at the $0 \%$ strain state by exposure to 10000 p.p.m. $\mathrm{CH}_{4}$ and 120 p.p.m. $\mathrm{CO}_{2}$ (Supplementary Figure S16). It was found that the sensor exhibited higher sensitivity to $\mathrm{CH}_{4}$ than $\mathrm{CO}_{2}$, illustrating its good selectivity. The tunable sensitivity of the gas sensor depending on the strain state is a
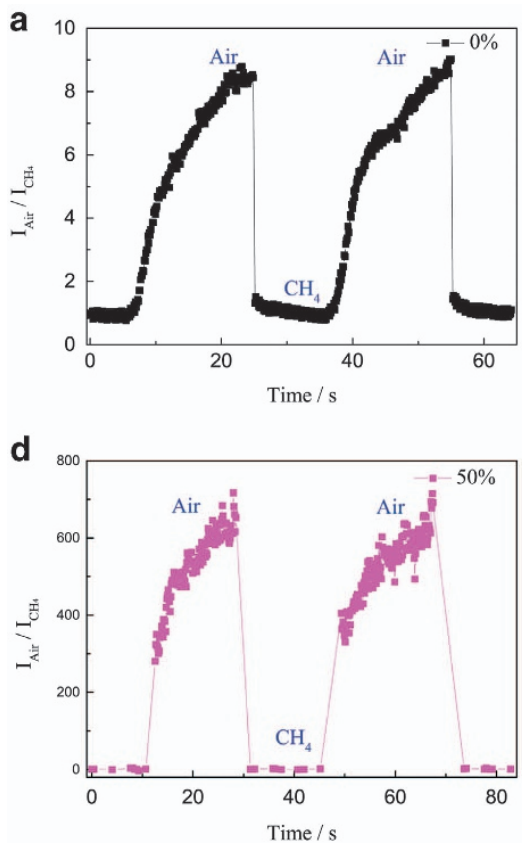

b

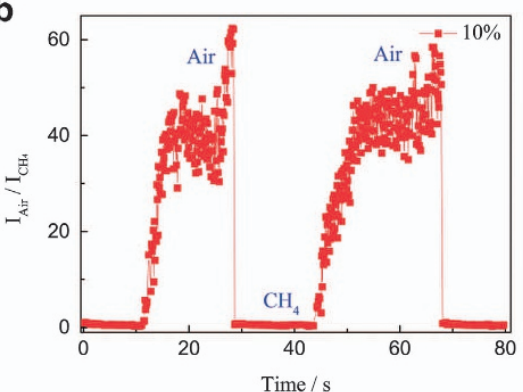

e

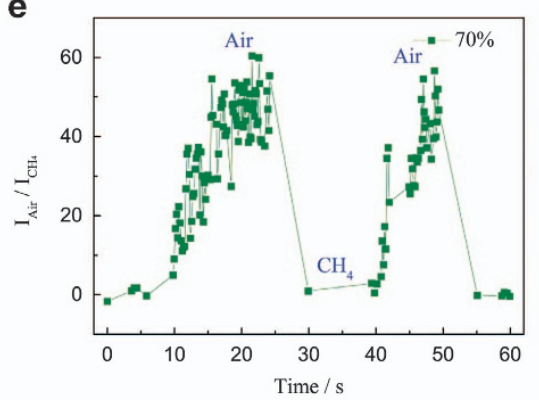

C

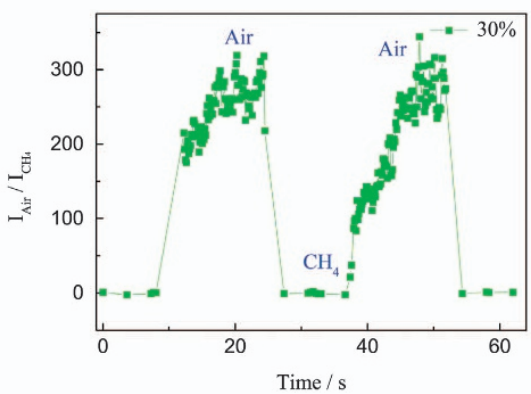

f

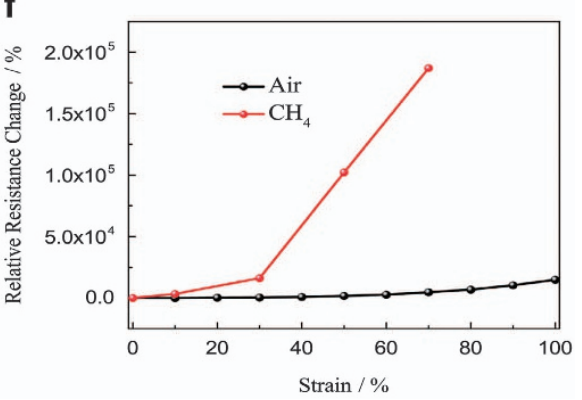

Figure 5 Time-dependent response and recovery curves of the PANI nanowire array-based sensor exposed to $\mathrm{CH}_{4}$ gas under different strains of (a) $0 \%$ strain, (b) $10 \%$ strain, (c) $30 \%$ strain, (d) $50 \%$ strain and (e) $70 \%$ strain. (f) Plots of the relative resistance change versus strain for the PANI nanowire array-based sensor exposed to air and $\mathrm{CH}_{4}$ gas. 
unique feature for adjustable, wearable gas-sensing e-skin, which can act as a gaseous multimeter with adjustable sensitivity by adjusting the strain state. The above results illustrate that our sensing platform has a similar functionality to snakes, which can simultaneously sense different tactile and olfactory stimuli. Although the corresponding output signals influence each other sometimes, this problem can be well resolved by rational design of the bionic component to differentiate multiple stimuli, such as through the combination of serial and parallel sensing channels in the device (similar to the sophisticated nervous system in snakes), depending on the specific application needs.

\section{CONCLUSIONS}

In summary, we report a bionic snake skin sensing platform composed of semitransparent PANI nanowire arrays on a thin PDMS elastic substrate that can simultaneously sense mechanical strain, pressure and $\mathrm{CH}_{4}$ gas. The snake skin-like PANI nanowire array-based strain sensor exhibits a large relative resistance change of $14897 \%$ at $100 \%$ strain. The highest gauge factor demonstrated in this work is 149 at $100 \%$ strain. The performance is significantly better than that of most conducting polymer-based sensors reported so far and is comparable to or even better than traditional metal and carbon nanowire/nanotubebased strain sensors. Moreover, the bionic snake skin sensing platform can be used to smell hazardous $\mathrm{CH}_{4}$ gas with high sensitivity and fast response and recovery under different strain states. We believe that our semitransparent stretchable sensing platform could be applied in a wide range of applications in robotics, treatment, electronic skin, wearable health-monitoring devices and bionic systems.

\section{CONFLICT OF INTEREST}

The authors declare no conflict of interest.

\section{ACKNOWLEDGEMENTS}

This research is supported by the NRF Investigatorship Award NRF-NRFI201605 and the NRF Competitive Research Programme NRF-CRP13-2014-02, which are supported by the National Research Foundation, Prime Minister's Office, Singapore.

\section{PUBLISHER'S NOTE}

Springer Nature remains neutral with regard to jurisdictional claims in published maps and institutional affiliations.

1 Rogers, J. A., Someya, T. \& Huang, Y. Materials and mechanics for stretchable electronics. Science 327, 1603-1607 (2010).

2 Cai, G. F., Wang, J. X. \& Lee, P. S. Next-generation multifunctional electrochromic devices. Acc. Chem. Res. 49, 1469-1476 (2016).

3 Lee, J., Yoon, J., Kim, H. G., Kang, S., Oh, W.-S., Algadi, H., Al-Sayari, S., Shong, B., Kim, S.-H., Kim, H., Lee, T. \& Lee, H.-B.-R. Highly conductive and flexible fiber for textile electronics obtained by extremely low-temperature atomic layer deposition of $\mathrm{Pt}$ NPG Asia Mater. 8, e331 (2016).

4 Lee, B. Y., Sung, M. G., Lee, H., Namgung, S., Park, S. Y., Choi, D. S. \& Hong, S. Integrated devices based on networks of nanotubes and nanowires. NPG Asia Mater. 2, 103-111 (2010).

5 Huynh, T.-P. \& Haick, H. Self-healing, fully functional, and multiparametric flexible sensing platform. Adv. Mater. 28, 138-143 (2016).

6 Xue, X., Fu, Y., Wang, Q., Xing, L. \& Zhang, Y. Outputting olfactory bionic electric impulse by PANI/PTFE/PANI sandwich nanostructures and their application as flexible, smelling electronic skin. Adv. Funct. Mater. 26, 3128-3138 (2016).

7 Lee, H.-B., Bae, C.-W., Duy, L. T., Sohn, I.-Y., Kim, D.-I., Song, Y.-J., Kim, Y.-J. \& Lee, N.-E. Mogul-patterned elastomeric substrate for stretchable electronics. Adv. Mater. 28, 3069-3077 (2016)

8 Kang, D., Pikhitsa, P. V., Choi, Y. W., Lee, C., Shin, S. S., Piao, L., Park, B., Suh, K.-Y., Kim, T.-I. \& Choi, M. Ultrasensitive mechanical crack-based sensor inspired by the spider sensory system. Nature 516, 222-226 (2014).
9 Roh, E., Hwang, B.-U., Kim, D., Kim, B.-Y. \& Lee, N.-E. Stretchable, transparent, ultrasensitive, and patchable strain sensor for human-machine interfaces comprising a nanohybrid of carbon nanotubes and conductive elastomers. ACS Nano 9, 6252-6261 (2015)

10 Yang, T., Li, X., Jiang, X., Lin, S., Lao, J., Shi, J., Zhen, Z., Li, Z. \& Zhu, H. Structural engineering of gold thin films with channel cracks for ultrasensitive strain sensing. Mater. Horiz. 3, 248-255 (2016).

11 Zhou, C., Bette, S. \& Schnakenberg, U. Flexible and stretchable gold microstructures on extra soft poly(dimethylsiloxane) substrates. Adv. Mater. 27, 6664-6669 (2015).

12 Song, E., Kang, B., Choi, H. H., Sin, D. H., Lee, H., Lee, W. H. \& Cho, K. Stretchable and transparent organic semiconducting thin film with conjugated polymer nanowires embedded in an elastomeric matrix. Adv. Electron. Mater. 2, 1500250 (2016).

13 Long, Y.-Z., Li, M.-M., Gu, C., Wan, M., Duvail, J.-L., Liu, Z. \& Fan, Z. Recent advances in synthesis, physical properties and applications of conducting polymer nanotubes and nanofibers. Prog. Polym. Sci. 36, 1415-1442 (2011).

14 Jang, J., Ha, J. \& Cho, J. Fabrication of water-dispersible polyaniline-poly(4-styrenesulfonate) nanoparticles for inkjet-printed chemical-sensor applications. Adv. Mater. 19, 1772-1775 (2007)

15 Bai, H. \& Shi, G. Gas sensors based on conducting polymers. Sensors 7, 267 (2007).

16 Yu, G.-F., Yan, X., Yu, M., Jia, M.-Y., Pan, W., He, X.-X., Han, W.-P., Zhang, Z.-M., Yu, L.-M. \& Long, Y.-Z. Patterned, highly stretchable and conductive nanofibrous PANI/ PVDF strain sensors based on electrospinning and in situ polymerization. Nanoscale $\mathbf{8}$, 2944-2950 (2016).

17 Ryu, H., Cho, S. J., Kim, B. \& Lim, G. A stretchable humidity sensor based on a wrinkled polyaniline nanostructure. RSC Adv. 4, 39767-39770 (2014).

18 Park, H., Jeong, Y. R., Yun, J., Hong, S. Y., Jin, S., Lee, S.-J., Zi, G. \& Ha, J. S. Stretchable array of highly sensitive pressure sensors consisting of polyaniline nanofibers and Au-coated polydimethylsiloxane micropillars. ACS Nano $\mathbf{9}$, 9974-9985 (2015).

19 Chiou, N.-R., Lu, C., Guan, J., Lee, L. J. \& Epstein, A. J. Growth and alignment of polyaniline nanofibres with superhydrophobic, superhydrophilic and other properties. Nat. Nano 2, 354-357 (2007).

20 Xu, J., Wang, K., Zu, S.-Z., Han, B.-H. \& Wei, Z. Hierarchical nanocomposites of polyaniline nanowire arrays on graphene oxide sheets with synergistic effect for energy storage. ACS Nano 4, 5019-5026 (2010).

21 Schnippering, M., Powell, H. V., Mackenzie, S.,R. \& Unwin, P. R. Real-time monitoring of polyaniline nanoparticle formation on surfaces. J. Phys. Chem. C 113, 20221-20227 (2009).

22 Cui, M. Q., Ng, W. S., Wang, X., Darmawan, P. \& Lee, P. S. Enhanced electrochromism with rapid growth layer-by-layer assembly of polyelectrolyte complexes. Adv. Funct. Mater. 25, 401-408 (2015).

23 MacDiarmid, A. G. 'Synthetic metals': a novel role for organic polymers (Nobel Lecture). Angew. Chem. Int. Ed. 40, 2581-2590 (2001).

24 Drury, A., Chaure, S., Kröll, M., Nicolosi, V., Chaure, N. \& Blau, W. J. Fabrication and characterization of silver/polyaniline composite nanowires in porous anodic alumina. Chem. Mater. 19, 4252-4258 (2007).

25 Wang, L., Chen, L., Yan, B., Wang, C., Zhu, F., Jiang, X., Chao, Y. \& Yang, G. In situ preparation of $\mathrm{SnO}_{2} @$ polyaniline nanocomposites and their synergetic structure for high-performance supercapacitors. J. Mater. Chem. A 2, 8334-8341 (2014).

26 Barlian, A. A., Park, W. T., Mallon, J. R., Rastegar, A. J. \& Pruitt, B. L. Review: semiconductor piezoresistance for microsystems. Proc. IEEE 97, 513-552 (2009).

27 Yamada, T., Hayamizu, Y., Yamamoto, Y., Yomogida, Y., Izadi-Najafabadi, A., Futaba, D. N. \& Hata, K. A stretchable carbon nanotube strain sensor for human-motion detection. Nat. Nano 6, 296-301 (2011).

28 Yan, C. Y., Wang, J. X., Kang, W. B., Cui, M. Q., Wang, X., Foo, C. Y., Chee, K. J. \& Lee, P. S. Highly stretchable piezoresistive graphene-nanocellulose nanopaper for strain sensors. Adv. Mater. 26, 2022-2027 (2014).

29 Amjadi, M., Pichitpajongkit, A., Lee, S., Ryu, S. \& Park, I. Highly stretchable and sensitive strain sensor based on silver nanowire-elastomer nanocomposite. ACS Nano 8, 5154-5163 (2014).

30 Cai, G. F., Wang, J. X., Qian, K., Chen, J. W., Li, S. H. \& Lee, P. S. Extremely stretchable strain sensors based on conductive self-healing dynamic cross-links hydrogels for human-motion detection. Adv. Sci. 4, 1600190 (2017).

31 Frutiger, A., Muth, J. T., Vogt, D. M., Mengüç, Y., Campo, A., Valentine, A. D., Walsh, C. J. \& Lewis, J. A. Capacitive soft strain sensors via multicore-shell fiber printing. Adv. Mater. 27, 2440-2446 (2015).

32 Liao, X., Liao, Q., Yan, X., Liang, Q., Si, H., Li, M., Wu, H., Cao, S. \& Zhang, Y. Flexible and highly sensitive strain sensors fabricated by pencil drawn for wearable monitor. Adv. Funct. Mater. 25, 2395-2401 (2015).

33 Pang, C., Lee, G.-Y., Kim, T.-i., Kim, S. M., Kim, H. N., Ahn, S.-H. \& Suh, K.-Y. A flexible and highly sensitive strain-gauge sensor using reversible interlocking of nanofibres. Nat. Mater. 11, 795-801 (2012).

34 Cheng, M. Y., Huang, X. H., Ma, C. W. \& Yang, Y. J. A flexible capacitive tactile sensing array with floating electrodes. J. Micromech. Microeng. 19, 115001 (2009).

35 Wu, Z., Chen, X., Zhu, S., Zhou, Z., Yao, Y., Quan, W. \& Liu, B. Room temperature methane sensor based on graphene nanosheets/polyaniline nanocomposite thin film. IEEE Sens. J. 13, 777-782 (2013).

36 Sattari, S., Reyhani, A., Khanlari, M. R., Khabazian, M. \& Heydari, H. Synthesize of polyaniline-multi walled carbon nanotubes composite on the glass and silicon substrates and methane gas sensing behavior of them at room temperature. J. Ind. Eng. Chem. 20, 1761-1764 (2014).

37 Yoshida, Y., Ota, Y., Eguchi, N., Kikuchi, N., Nobuta, K., Tran, H., Morino, I. \& Yokota, T. Retrieval algorithm for $\mathrm{CO}_{2}$ and $\mathrm{CH}_{4}$ column abundances from short-wavelength 
infrared spectral observations by the Greenhouse gases observing satellite. Atmos. Meas. Tech. 4, 717-734 (2011).

38 Zhang, Y., Kim, J. J., Chen, D., Tuller, H. L. \& Rutledge, G. C. Electrospun polyaniline fibers as highly sensitive room temperature chemiresistive sensors for ammonia and nitrogen dioxide gases. Adv. Funct. Mater. 24, 4005-4014 (2014).

39 Virji, S., Huang, J., Kaner, R. B. \& Weiller, B. H. Polyaniline nanofiber gas sensors: examination of response mechanisms. Nano Lett. 4, 491-496 (2004).

40 Wang, J., Chan, S., Carlson, R. R., Luo, Y., Ge, G., Ries, R. S., Heath, J. R. \& Tseng, H.-R. Electrochemically fabricated polyaniline nanoframework electrode junctions that function as resistive sensors. Nano Lett. 4, 1693-1697 (2004).

41 Brie, M., Turcu, R., Neamtu, C. \& Pruneanu, S. The effect of initial conductivity and doping anions on gas sensitivity of conducting polypyrrole films to $\mathrm{NH}_{3}$. Sens. Actuators B Chem. 37, 119-122 (1996). (c) (i) This work is licensed under a Creative Commons Attribution 4.0 International License. The images or other third party material in this article are included in the article's Creative Commons license, unless indicated otherwise in the credit line; if the material is not included under the Creative Commons license, users will need to obtain permission from the license holder to reproduce the material. To view a copy of this license, visit http:// creativecommons.org/licenses/by/4.0/

(C) The Author(s) 2017

Supplementary Information accompanies the paper on the NPG Asia Materials website (http://www.nature.com/am) 